\title{
CRYSTAL STRUCTURE AND DEFECTS IN NITROGEN-DEFICIENT GaN
}

\author{
S. Oktyabrsky,* K. Dovidenko, A. K. Sharma, V. Joshkin** and J. Narayan \\ Center for Advanced Materials and Smart Structures, North Carolina State University, Raleigh, \\ NC 27695, \\ * NYS Center for Advanced Technology, State University of New York at Albany, Albany, NY \\ 12203 , \\ ** Department of Electrical and Computer Engineering, University of Wisconsin, Madison, WI, \\ 53706
}

Cite this article as : MRS Internet J. Nitride Semicond. Res. 4S1, G6.43 (1999)

\begin{abstract}
$\underline{\text { Abstract }}$
We have studied crystal structure and associated defects in GaN films grown on sapphire under nitrogen-deficient conditions by metalorganic chemical vapor deposition (MOCVD) and pulsed laser deposition (PLD). The structural quality of the PLD films grown at $750{ }^{\circ} \mathrm{C}$ was comparable with those grown by MOCVD at $1050{ }^{\circ} \mathrm{C}$ having threading dislocations density of about $10^{10}$ $\mathrm{cm}^{-2}$ at a film thickness 150-200 nm. Microstructure of the PLD films grown at temperatures above $780^{\circ} \mathrm{C}$ was found to be similar to that of nitrogen-deficient MOCVD films indicating the loss of nitrogen due to thermal decomposition of the nitride layers. Nitrogen-deficient MOCVD and PLD films exhibit polycrystalline structure with a mixture of cubic zinc-blende and wurtzite hexagonal GaN grains retaining tetragonal bonding across the boundaries and hence the epitaxial orientations and polarity. Renucleation of the wurtzite phase at different $\{111\}$ planes of cubic $\mathrm{GaN}$ results in a rough and faceted surface of the film. Most of the stoichiometric films displayed (0001) Ga-face polarity, but the renucleated inclined wurtzite grains grew in the opposite $\mathrm{N}$-face polarity. The major defects related to the cubic structural metastability are stacking faults and microtwins which being nuclei of the metastable cubic phase have an extremely low energy. We elucidate that the cubic phase is more stable under the nitrogen deficiency and, therefore, can exist without decomposition at higher nitrogen vacancy concentrations in the material.
\end{abstract}

\section{Introduction}

The influence of critical growth parameters such as V/III ratio and substrate temperature on the surface morphology, electrical and optical properties of $\mathrm{GaN}$ films has been studied extensively [1-6]. In many cases, however, the obtained results are specific for particular growth methods and do not provide the insight into general tendencies of the growth process. Therefore, it is important to compare the major characteristics of the films grown by different processing methods. In this paper, we will show the influence of nitrogen deficiency on the microstructure and typical defects in the films grown by atmospheric pressure metalorganic chemical vapor deposition (MOCVD) and pulsed laser deposition (PLD) techniques. Compared to MOCVD and MBE methods, the PLD is highly nonequilibrium method. It is known to deliver more energetic (10-100 times higher than MBE) species to the substrate. The stoichiometry of the deposited films is one of the important advantages of the PLD. Due to congruent ablation of the target and close proximity of the substrate, the PLD transfers the composition of the target to the substrate almost without change of the stoichiometry. In the case of PLD of group III-nitrides, the stoichiometry is affected by two major factors: firstly, a very big difference in partial pressures of the components: atomic nitrogen and metal, and secondly, formation of stable nitrogen 
molecules which reduces atomic nitrogen concentration. Therefore, special efforts should be made to increase nitrogen incorporation into the growing layer. In the conventional processing methods for III-N growth, MOCVD and MBE, the special means for atomic nitrogen generation are utilized, such as high-temperature pyrolisis of ammonia in MOCVD, or plasma nitrogen sources in MBE. In the case of laser ablation, it is in principle possible to transfer the composition from the stoichiometric target to the substrate. In general, raising the growth temperature is effective in improving the crystallinity and purity of epitaxial films unless it causes thermal decomposition of the crystal, which occur in $\mathrm{GaN}$ above $600{ }^{0} \mathrm{C}$. Therefore, it is important to determine the growth temperature range in which thermal decomposition does not limit the properties of the nitrides. In this letter, we will focus on structural properties of $\mathrm{GaN}$ films grown by MOCVD and PLD under nitrogen-deficient conditions.

\section{$\underline{\text { Experimental Details }}$}

To study the influence of the stoichiometry on the film microstructure we used GaN films grown by MOCVD or pulsed laser deposition (PLD). These methods have very different thermodynamic and kinetic parameters, and, therefore, comparison of the results is crucial for both studying growth-property correlations as well as understanding the mechanisms of defect formation under nonstoichiometric conditions. Atmospheric pressure vertical MOCVD reactor with a mixture of trimethylgallium, ammonia and nitrogen as a carrier gas was used to grow $\mathrm{GaN}$ films on $\alpha-\mathrm{Al}_{2} \mathrm{O}_{3}$ (0001) substrates. The details of growth can be found elsewhere [7]. The growth process included nitridation, growth of a low-temperature AlN buffer layers and GaN films. The latter films were deposited at $950{ }^{\circ} \mathrm{C}$ with an average V/III mole ratio of 1000 . We have also employed PLD to grow $\mathrm{GaN}$ on $\alpha-\mathrm{Al}_{2} \mathrm{O}_{3}$ (0001). We used $\mathrm{KrF}$ excimer laser (wavelength - $248 \mathrm{~nm}$, pulse frequency - 10-15 Hz, pulse duration $20 \mathrm{~ns}$ ) to ablate a stoichiometric hot-pressed target. Target-substrate distance of $4.5 \mathrm{~cm}$, energy density of 3-4 $\mathrm{J} / \mathrm{cm}^{2}$ were used to prepare $150-250 \mathrm{~nm}$ - thick films. The films were deposited at a substrate temperature of $720-800{ }^{0} \mathrm{C}$ in a vacuum chamber with a base pressure of $5 \times 10^{-7}$ Torr on solvent cleaned substrates. The details of the target and substrate preparation, their characteristics and parameters of PLD are summarized elsewhere [8]. It should be emphasized that the PLD GaN films are much thinner $(<0.3 \mu \mathrm{m})$ than MOCVD films $(>0.5 \mu \mathrm{m})$.. The characterization of defects and interfaces in the films was carried out using transmission electron microscopy (TEM). A $200 \mathrm{keV}$ Topcon 002B microscope with a point-to-point resolution of $1.8 \AA$ was used for this study. The cross-sectional TEM samples were prepared by mechanical polishing followed by dimpling to a thickness of approximately 15-25 $\mu \mathrm{m}$ with final thinning using Ar-ion milling at $5 \mathrm{kV}$. Convergent beam electron diffraction (CBED) patterns were obtained using a focused probe of $10-15 \mathrm{~nm}$ with a convergence semiangle of $2 \mathrm{mrad}$.

\section{$\underline{\text { Results }}$}

Fig. 1 shows cross-sectional micrographs of two GaN samples grown by MOCVD in the same run at the same substrate temperature of $950{ }^{\circ} \mathrm{C}$. The sample shown in the Fig. 1(a) was placed close to the metalorganic jet in the MOCVD reactor and consequently was grown under ammonia- (or nitrogen-) deficient conditions. The film in the Fig. 1(b) was placed away from the metalorganic stream and grown under group III-deficient conditions. The film (b) was thinner than the film (a) indicating that the film (b) was also grown far from stoichiometric surface conditions, but in N-rich conditions. We have estimated the effective mole ratio of the components using growth rate vs. ratio calibration curves. The films in Figs. 1(a) and 1(b) have been grown at the V/III molar ratios of approximately 2000 and 500, respectively. The film 

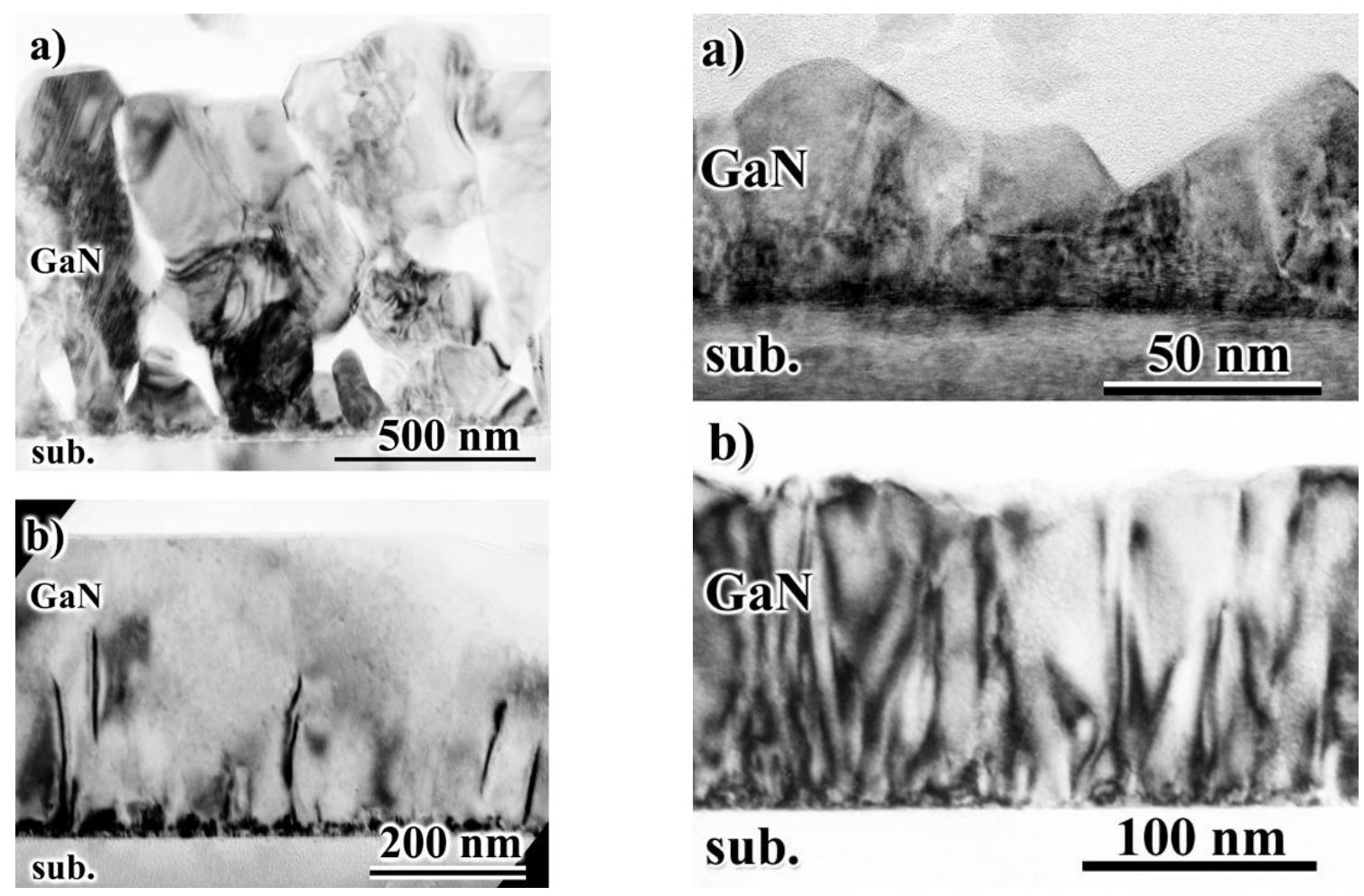

Fig. 1. TEM cross-sectional micrographs of the MOCVD-grown GaN at Ga-rich (a) and N-rich (b) conditions.

Fig. 2. TEM cross-sectional micrographs of the PLD-grown GaN films at $750{ }^{\circ} \mathrm{C}$ (a) and $780{ }^{\circ} \mathrm{C}$ (b).

grown under $\mathrm{N}$-rich conditions has mirror-like surface and is very uniform, showing only tiny $(\sim 10 \mathrm{~nm})$ pits at the dislocations and/or inversion domain boundaries, unlike the $\mathrm{N}$-deficient film which looks hazy. The TEM image of this $\mathrm{N}$-deficient film shows a very rough faceted surface with a hill-to-valley roughness of about $150 \mathrm{~nm}$. This layer contains also misoriented grain and large pores between the grains.

Fig. 2 presents the images of GaN films grown by PLD. The major difference of these two films is the deposition temperature. The film in The Fig. 2(a) was grown at $780^{\circ} \mathrm{C}$ while the one in The Fig.2(b) was grown at $750{ }^{\circ} \mathrm{C}$. The film (a) exhibits very rough faceted surface with misoriented grains similar to the morphology presented in the Fig. 1(a). Note that the PLD films in the Fig. 2 are thinner than MOCVD films shown in the Fig. 1. The structural quality of the PLD film grown at $750{ }^{\circ} \mathrm{C}$ (Fig. 2b) is comparable to those grown by MOCVD in the temperature range $950-1050{ }^{\circ} \mathrm{C}$. The PLD film at lower temperature (Fig. 2(b)) displays a typical columnar structure consisting of slightly misoriented subgrains bounded by threading dislocations with a density of about $2 \cdot 10^{10} \mathrm{~cm}^{-3}$ at a film thickness $150-200 \mathrm{~nm}$ as measured from plan-view TEM micrographs [9]. The threading dislocation density decreases with the thickness of the film, and it is quite close (factor 3-5 higher) to that for high-quality GaN films with a similar thickness. We show in the following that the PLD films grown at higher temperature are likely to be nitrogen-deficient.

The micrographs in Figs. 3(a) and 3(b) show the higher magnification cross-sectional images of GaN films grown by MOCVD with deficient ammonia flow and by PLD at $780^{\circ} \mathrm{C}$, respectively. The major microstructural feature of the both films is the mixture of the wurtzite (hexagonal) and zinc-blende (cubic) phases. The presence of the grains of the cubic phase in the films is illustrated by selected area electron diffraction in the right inset of the Fig. 3(a) (MOCVD film), and the convergent beam electron diffraction (CBED) in the inset of the Fig. 3(b) (thin PLD film). The cubic structure has four equivalent families of close-packed $\{111\}$ 

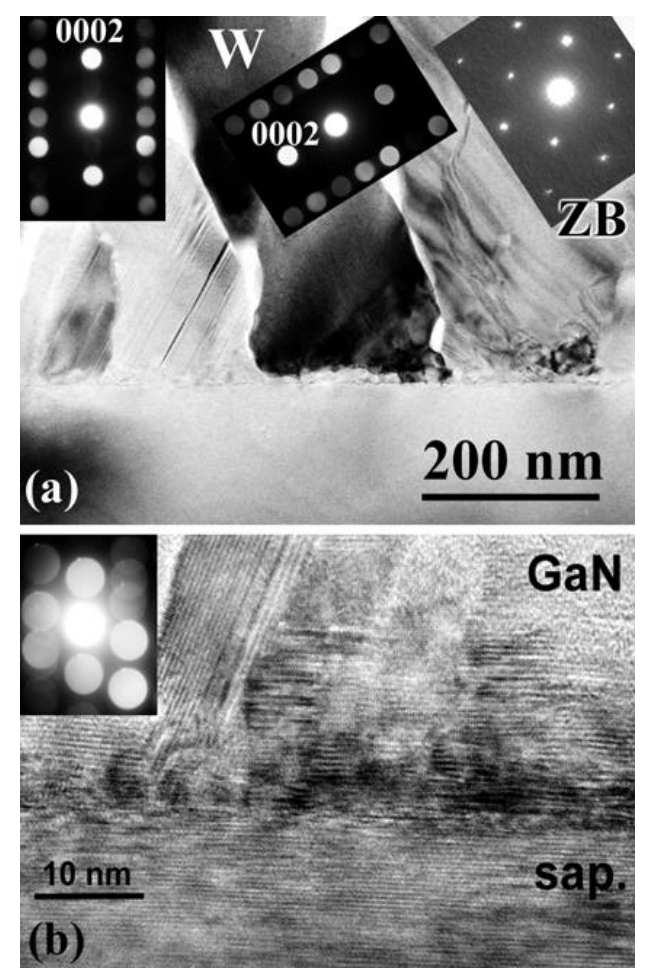

Fig.3. TEM images in $[11 \overline{2} 0]$ projection of the $\mathrm{GaN}$ films: (a) Ndeficient MOCVD showing both wurtzite (W) and zinc-blende (ZB) grains; the insets: ED patterns from a wurtzite grain oriented with caxis normal to the surface (not shown in the image), from misoriented "W"-grain, and "ZB"-grain;

(b) grown by PLD at $780^{\circ} \mathrm{C}$ showing the mixture of wurtzite and zinc-blende $\mathrm{GaN}$ phases; the inset: micro-diffraction pattern of a small GaN grain with cubic symmetry in a (110) zone.

planes while the hexagonal structure has a single (0001) close-packed plane orientation. Two possible cubic orientations can nucleate on the (0001) hexagonal surface if the tetrahedral bonding is preserved across the interface. These orientations are schematically shown in Figs. 4(a) and (b). The tetrahedron of $\{111\}$ planes is highlighted by the dashed lines for each orientation. Consequently, there are seven different orientations of the close-packed planes (one plane is common for both tetrahedra) in the cubic grains grown epitaxially on wurtzite structure. The growth of cubic zinc-blende phase can be stabilized during growth and large grains can be formed such as indicated by "ZB" in the Fig. 3(a). Otherwise, the wurtzite phase can renucleate with the basal plane parallel to on one of the seven $\{111\}$ faces of the cubic grains with the crystallographic relationships $(0001) / /\{111\}$, and $\langle 11 \overline{2} 0\rangle / /<1 \overline{1} 0\rangle$. Indeed, as clearly seen in the Figs. 3(a) and (b), the resultant microstructure of the film exhibits grains of cubic phase and large wurtzite grains misoriented by approximately $70^{\circ}$ corresponding to the angle between $\{111\}$ planes $\left(70.5^{0}\right)$ of the cubic structure. Due to

(a)
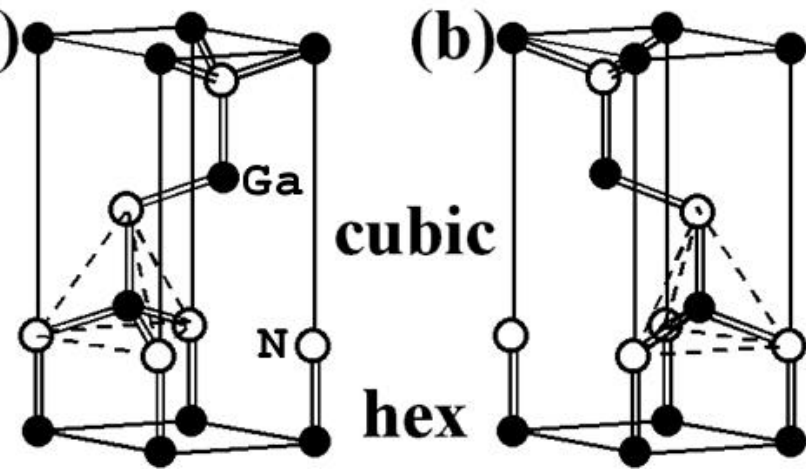

Fig. 4. Two possible orientations of zinc-blende cubic phase that can nucleate on the (0001) hexagonal wurtzite surface.

growth of these grains in different directions, the resulting film surface is very rough and faceted. The films, therefore, are polycrystalline but contain certain orientations of the phases. In addition, as deduced from the CBED patterns (Fig.3(a)-insets), the wurzite grains have different polarity depending on orientation (see below), that also provides the evidence for the epitaxial origin of wurtzite/zinc-blende interfaces.

\section{$\underline{\text { Disscusion }}$}

The formation of the metastable zinc-blende GaN phase is most likely due to nonstoichiometry of the growth conditions. They result from the loss of nitrogen at a higher temperature in the PLD film or from nitrogen-deficient conditions in the MOCVD film. We envision that the cubic phase is more stable under the nitrogen deficiency and, therefore, can 
exist without decomposition at much higher nitrogen vacancy concentrations in the material. It is worth comparing these results with the common procedures of cubic GaN growth. A wellestablished way to form the zinc-blende $\mathrm{GaN}$ is the growth on a substrate with cubic symmetry such as GaAs(001) [3-6]. The crystal structure of the GaN grown on GaAs can be controlled by changing V/III ratio in plasma-assisted MBE [4] or in low-pressure RF-plasma assisted MOCVD [5] techniques. The cubic GaN was formed under N-deficient conditions, and the hexagonal GaN was primarily grown under $\mathrm{N}$-rich conditions. It was also shown [6] that the crystalline and optical quality of zinc-blende GaN film grown by conventional low-pressure MOCVD improved at low V/III ratios. These results also correlate with the enhanced stability of the cubic phase at lower V/III ratios.

The major defects related to the cubic structural metastability are stacking faults and microtwins which can be seen in the N-deficient films in the Figs. 1 through 3. These planar defects lie in the close-packed (0001) hexagonal and $\{111\}$ cubic planes, and being nuclei of the metastable cubic phase these faults have an extremely low energy. A similar crystal structure of the GaN films was reported in a detailed study of the microstructure of the nucleation layer grown by MOCVD at $600{ }^{0} \mathrm{C}$ [10]. As-grown $20 \mathrm{~nm}$ thick layers exhibited cubic nature predominantly, and were converted into hexagonal upon exposure to $1080{ }^{\circ} \mathrm{C}$ annealing under ammonia flow. Formation of the cubic $\mathrm{GaN}$ at low temperature can also be the result of the nitrogen deficiency because of insufficient pyrolisis of ammonia at low substrate temperatures. However, another effect of low temperature growth, namely the increase of stacking disorder due to slower adatom mobility, cannot be ruled out. In any case, nonstoichiometric nitrogen-deficient surface promotes the shift of the equilibrium towards the cubic phase and (even if it is not the primary factor) stabilizes the growth of the cubic GaN.

As can be clearly seen from the Fig. 4, the hexagonal grains with c-axis normal to the substrate surface and those with the inclined c-axis would have opposite face polarity, if the latter grains are nucleated on the cubic phase faces and the polarity is preserved across the phase interface. We have used CBED to determine the polarity of the grains [11]. Due to dynamic electron beam scattering by the non-centrosymmetric specimen, the CBED pattern loses the inversion symmetry. Unlike a common method involving simulation of the CBED contarst for quite thick specimen regions [12,13], we have proposed a technique based on the study of the thinnest $(<150 \AA$ ) regions of the wurtzite grains [11]. The simulaton of the CBED as well as pendellössung curves (beam intensity vs. thickness) shows that the pattern from a very thin regions carries unambiguous information about polarity as can be seen in the inserts of the Fig. 3(a). In [11 $\overline{2} 0]$ orientation for the thinnest sections, the beams (0002), $(1 \overline{1} 0 \overline{2})$ and $(\overline{1} 10 \overline{2})$ are stronger than $(000 \overline{2}),(\overline{1} 102)$ and $(1 \overline{1} 02)$, respectively, giving a fingerprint for the polarity determination. In this case, the [0002] direction aims from $\mathrm{Ga}$ to $\mathrm{N}$ in the wurtzite structure which corresponds to the Ga-polarity of the (0002) surface.

The smooth films grown under optimum or nitrogen-rich conditions by MOCVD and at optimum temperature by PLD have shown Ga-face (0001) polarity [11]. The same Ga-polarity has been found for the wurtzite grains with c-axis normal to the substrate surface as shown in the left inset of the Fig. 3(a). In the grains with the inclined c-planes, the outer surface correspond to $\mathrm{N}$-face (right inset of the Fig. 3(a)) in agreement with the scheme in the Fig. 4.

The polarity of the growing film depends upon several factors including the parameters of nitridation step, recrystallization of low temperature buffer layer and the kinetics of growth of two polarities [14]. A detailed study of the surface morphology of the GaN films with the different polarities [15] has revealed that the Ga-face (0001) surface is much smoother than the surface with N-polarity $(000 \overline{1})$. Therefore, films with Ga-polarity are preferable for applications. Most of the GaN films (both PLD and MOCVD grown) that we have investigated, had the Gapolarity of the (0002) planes, some of them showed the presence of columnar inversion domains 
[9]. Applying the present study to the initially polycrystalline low-temperature grown buffer layers, it should be noted, that the polarity of the primary orientation of the epitaxial GaN grains cannot be altered by the cubic phase formation (Fig. 4). However, the grains of the wurtzite phase with inclined c-axis will have the opposite polarity and under particular growth conditions can attain higher growth rates and become dominant. This mechanism will contribute to the roughness and defective microstructure of the GaN films.

\section{Conclusions}

In conclusion, we have shown that nitrogen-deficient growth of $\mathrm{GaN}$ results in formation of grains with cubic zinc-blende structure. Renucleation of the wurtzite phase at different $\{111\}$ planes of cubic GaN leads to polycrystalline structure of the film with rough and faceted surface. Grains having cubic or hexagonal structure exhibit epitaxial relationships and preserve polarity of the Ga-N bonds across the interfaces. We elucidate that the cubic phase is more stable under the nitrogen deficiency and, therefore, can exist without decomposition at higher nitrogen vacancy concentrations in the material.

\section{$\underline{\text { Acknowledgments }}$}

The work was supported by National Science Foundation through the Center for Advanced Materials and Smart Structures.

\section{$\underline{\text { References }}$}

1. R. Held, D.E. Crawford, A.M. Johnston, A.M. Dabiran, and P.I.Cohen, J. Electronic Mat. 26, 272 (1997).

2. O. Briot, J.P. Alexis, S. Sanchez, B. Gil, and R.L. Aulombard, Solid-State Electronics, 41, 315 (1997).

3. D. Chandrasekhar, D.J.Smith, S. Strite, M.E. Lin, and H. Morcoç, J. Crystal Growth, 152, 135 (1995).

4. O. Brandt, H. Yang, B. Jenichen, Y. Suzuki, L. Deritz, and K.H. Ploog, Phys. Rev., B 52, R2253 (1995) .

5. M. Sato, J. Appl. Phys., 78, 2123 (1995).

6. A. Nakadaira and H. Tanaka, J. Electronic Mat., 26, 320 (1997).

7. V.A. Joshkin, J.C. Roberts, F.G. McIntosh, S.M. Bedair, E.L. Piner, and M.K. Behbehani, Appl. Phys. Lett., 71, 234 (1997).

8. S. Oktyabrsky, K. Dovidenko, A.K. Sharma, J. Narayan, in Advances of Laser Ablation of Materials, edited by R. Singh, D. Lowndes, D. Chrisey, J. Narayan, T. Kawai, E. Fogarassy, (Mater. Res. Soc. Proc. 526, San Francisco, 1988) pp.287-292.

9. K. Dovidenko, S. Oktyabrsky, J. Narayan, V. Joshkin, M. Razeghi, in Nitride Semiconductors, edited by F.A. Ponce, S.P. DenBaars, B.K. Meyer, S., Nakamura, S. Strite, (Mater. Res. Soc. Proc. 482, Boston, 1997) pp. 411-416.

10. X.H.Wu, D. Kapolnek, E.J. Tarsa, B. Heying, S. Keller, B.P. Keller, U.K. Misra, S.P. DenBaars, and J.S. Speck, Appl. Phys. Lett., 68, 1371 (1996).

11. K. Dovidenko, S. Oktyabrsky, J. Narayan, and M. Razeghi, MRS Internet J. Nitride Semicond. Res. 4S1, G6.46 (1999).

12. Z. Liliental-Weber, C. Kisielowski, S. Ruvimov, Y. Chen, J. Washburn, I. Grzegory, M.Bockowski, J. Jun, and S. Porowski, J. Electron. Mat., 25, 1545 (1996).

13. F.A. Ponce, D.P.Bour, W.T. Young, M. Saunders, and J.W. Steeds, Appl. Phys. Lett., 69337 (1996).

14. J.L. Rouviere, M. Arlery, R. Niebuhr, K.H. Bachem, and O. Briot, Mat. Sci. Eng., B43, 161 (1997)

15. M. Seelmann-Eggebert, J.L Weyher, H. Obloh, H. Zimmermann, A. Rar, and S. Porowski, Appl. Phys. Lett., 71, 2635 (1997). 